Die entlang dieser Dimensionen aufschlüsselbaren Identitätsthemen sind für Wegener auch in der Medienaneignung sowie den Motiven der Zuwendung zu medialen Bezugspersonen eine relevante Größe: Identitätsthemen „stellen eine zentrale Frage individueller Konstitution in den Mittelpunkt der Medienaneignung" (72). Mit ihrem subjektorientierten Zugriff reproduziert Wegener in ihren Ergebnissen vielfach jedoch das, was sie an den Identitätstheorien im zweiten Kapitel selbst kritisiert, ihre Unbestimmtheit angesichts medialer Bezüge bzw. Aneignungsweisen. Lässt sich dieser Widerspruch an dieser Stelle aber als Kritikpunkt aufrechterhalten, wenn aus der subjektorientierten Perspektive angesichts der unterstellten Pluralisierung von Lebenswelten kaum ein anderer Weg zu bleiben scheint, als zunächst einmal Identitätsprojekte in ihrem Medienbezug zu durchdringen und darzustellen?

Die relevanten Ergebnisse der Studie liegen v. a. in der Ausleuchtung der Handlungsräume, in denen sich die Sozialisation Jugendlicher aktuell vollzieht. Dabei kommt es Wegener auf die „Transformationen, Verfremdungen und Neugestaltung entsprechender Vorlagen in realen und imaginären Prozessen“ (49) bei der Aneignung medialer Bezugspersonen an. An dieser Stelle findet sich eine Fülle an umfassend aufgearbeiteten und belegten Befunden zu den Formen und Motiven der Zuwendung zu medialen Bezugspersonen, vor den Hintergrund der Bewältigung von Lebensaufgaben, die auch für die Rezeptionsforschung interessante Perspektiven eröffnen. Zweifelsohne ist der Blick auf die Folgen pluraler Lebenswelten zu richten und zweifelsohne finden sich hier Identitätsthemen, bei deren Bewältigung die Medienpädagogik unterstützend eingreifen kann. Dass die "Wahrnehmung von Medienhandeln als subjektiver Aneignung und das Verstehen individueller Deutung“ (386) zum Grundlagenwissen der Medienpädagogik gehören müssen, kann jedoch vor dem Hintergrund des interdisziplinären Anspruchs der Studie nicht darüber hinwegführen, dass grundlegende Fragen zur Subjektkonstitution unbeantwortet bleiben. Diese erscheinen mir erst dann differenzierter behandelbar, wenn eine generalisierende Perspektive angestrebt und nicht wie von Wegener, zurückgewiesen wird.

Udo Göttlich

\section{Dietrich Westphal}

\section{Föderale Privatrundfunkaufsicht im demo- kratischen Verfassungsstaat}

Verwaltungs- und verfassungsrechtliche

Analyse der Kommission zur Ermittlung der

Konzentration im Medienbereich (KEK)

Berlin: Duncker \& Humblot, 2007. - 664 S.

(Schriften zum Öffentlichen Recht; 1072)

ISBN 978-3-428-12318-6

Zugl.: München, Univ., Diss., 2005/2006

Die über 600 Seiten umfassende Dissertation gilt einer Institution, deren Bedeutung rechts- und verwaltungswissenschaftlich längst noch nicht ausgelotet ist. In drei unterschiedlich großen Hauptteilen beleuchtet der Autor die Entstehungsgeschichte der KEK und ihre verfassungs- und europarechtlichen Rahmensetzungen, unternimmt eine verwaltungsrechtliche Beschreibung und Einordnung der KEK und analysiert schließlich die verfassungsrechtlichen Vorgaben. Die Arbeit nimmt dabei weniger die spezifisch medienrechtlichen Bezüge auf und sucht stärker den Anschluss an die Dogmatik des allgemeinen Verwaltungs- und Verfassungsorganisationsrechts.

Die Hälfte der Arbeit (S. 140-450) ist der „Rechtsstruktur der KEK“ gewidmet. Nach einer knappen Zusammenfassung der rechtlichen Grundlagen erörtert der Autor in großer Ausführlichkeit und unter Behandlung aller bekannten Rechtsfragen die Aufgaben und Instrumente. Die aufgeworfenen Fragen werden entlang der einzelnen gesetzlichen Tatbestandsmerkmale und unter umfassender Heranziehung der Literatur und Rechtsprechung wie auch der Entscheidungspraxis der KEK aufgearbeitet.

Das sich anschließende Kapitel zur verwaltungsorganisationsrechtlichen Klassifizierung der KEK (S. 290-360) darf als das interessanteste des Buches gelten. Westphal sucht hierin die KEK in den Kanon der Organisationsformen einzuordnen. Er behandelt die in der Literatur bislang nicht vertieft behandelte Frage nach der „Organ“-Qualität der KEK und begründet mit vielen guten Argumenten, warum sich die Einrichtung diesem Organisationstyp - versteht man ihn klassisch - nicht zuordnen lassen will. Nach richtiger Verneinung des Behördencharakters (und weiterer Zuordnungen) klassifiziert er die KEK als Organisationstyp 
eigener Art, und zwar als gemeinsame Einrichtung der Länder mit spezifischen Merkmalen der Kollegialität, Zentralität und funktionellen Verselbstständigung. Hier leistet der Autor einen wichtigen Beitrag zur dogmatischen Klärung der Organisationsstruktur der KEK. Bei der organisationsrechtlichen Typisierung bleibt die Arbeit aber leider stehen. Weder wird sie für die absehbaren Veränderungen der länderübergreifenden Aufsichtsstrukturen im privaten Rundfunk noch für die verwaltungsrechtliche Typenbildung fruchtbar gemacht. Da die KEK isoliert und nicht in ihrer Einpassung in die Gesamtstruktur der Medienaufsicht behandelt wird, bleibt unreflektiert, dass die zentrale Medienaufsicht mit den vom Autor beschriebenen Strukturelementen schon seit einiger Zeit Konturen ausbildet, die in Richtung einer Regulierungsbehörde weisen. Verfassungsrechtliche Anschlussfragen zu Staatsferne und demokratischer Legitimation, die die Strukturbeschreibung geradezu herausfordert, werden später zwar behandelt, bleiben aber ohne konkreten und detaillierten Bezug zu den ausführlichen Aufgabenbeschreibungen und organisationsrechtlichen Ausführungen.

Die Ausführungen zum Verhältnis von KEK und KDLM sind mit dem Zehnten Rundfunkänderungsstaatsvertrag zwar nur noch von historischem Wert, bleiben aber von verwaltungs(rechts)wissenschaftlichem Interesse. Obwohl die Aufgabenstellung ausdrücklich nur die KEK umfasst, bezieht der Autor konsequent und zu Recht auch die Organisation der KDLM in seine Untersuchung ein. Hier beschränkt sich das Buch allerdings auf die verwaltungsrechtliche Klassifizierung und lässt verfassungsrechtliche Fragen nach der Pluralität und demokratischen Legitimation des Gremiums, auch als Institution der gesamten Medienaufsicht, offen. Die Einordnung als nichtständige Konferenz der Länder (S. 392397), mag sie auch überzeugend sein, bleibt damit folgenlos.

In den übrigen Teilen der Arbeit werden alle bekannten, sich um die KEK rankenden Themen angesprochen und ausführlich bearbeitet. Der erste Teil zu den historischen Grundlagen und Rahmenaspekten nimmt viele allgemeine Fragen auf, etwa die zur „dienenden Rundfunkfreiheit“ (S. 94ff.) oder zum Verhältnis von Medien- und Wirtschaftsrecht (S. 111ff.). Mit dem Kapitel zur „Vielfaltssicherung in Europa“ (S. 122ff.) zeigt der Autor ein spezifisches europarechtliches Interesse, dem seltene Ausführungen zur „European Platform of Regulatory Authorities“ (EPRA) zu verdanken sind. Die Forderung, von deutscher Seite die KEK an der EPRA zu beteiligen, könnte im Lichte der durch den Zehnten Rundfunkänderungsstaatsvertrag modifizierten Aufsichtsstrukturen neue Aktualität gewinnen.

Der dritte, verfassungsrechtliche Hauptteil (S. 451-607) behandelt bekannte Fragen der Staatsferne und Pluralität (die präzise unterschieden werden), die Fragen demokratischer Legitimation sowie die Zulässigkeit der zentralen Aufsichtstruktur mit Blick auf das Bundesstaatsprinzip. Die Ausführungen sind im Gegensatz zu dem höchst detaillierten verwaltungsrechtlichen Kapitel mit größerem Pinsel gezeichnet und damit leichter lesbar. Der Autor leitet mit Ausführungen zur Staatsferne ein, die weder in den Argumenten noch im Ergebnis überzeugen können. $\mathrm{Zu}$ verkürzend konzentriert er sich auf das Besetzungsverfahren und vernachlässigt die Anforderungen an die Qualifizierung und die besondere Rechtsstellung der KEK-Mitglieder. Dies ist umso erstaunlicher, als im späteren Kapitel zur demokratischen Legitimation einerseits deren Bezüge zum Gebot der Staatsferne gesehen werden und andererseits die Weisungsfreiheit der KEK-Mitglieder diskutiert wird (S. 514ff.). Der Vorschlag, die KEK von den Landesmedienanstalten wählen zu lassen, ist zwar nicht unoriginell, bleibt aber im Lichte des (doch ebenfalls verfassungsrechtlichen) Gebots einer effektiven Rundfunkaufsicht fragwürdig.

Schon im Vorwort lässt sich erkennen, dass ein besonderes Augenmerk des Autors der Behandlung demokratischer Legitimationsfragen gilt. Das Buch arbeitet zunächst den tradierten Ansatz heraus, der auf die funktionelle und institutionelle, die organisatorisch-personelle und die sachlich-inhaltliche Legitimationsform abhebt. Anschließend referiert er die Alternativmodelle einer aus der funktionalen Selbstverwaltung heraus begründeten autonomen Legitimation, einer „kollektiven personellen Legitimation" und eines stärker am Aufgabenbezug der Verwaltungstätigkeit ausgerichteten Legitimationskonzepts. Die KEK, die ganz im Zentrum der verwaltungsorganisationsrechtlichen Ausführungen gestanden hatte, bildet hier eher Anlass und konkreten Anwendungsfall einer weiter ausgreifenden Fragestellung. Stärker gerät sie erst wieder in den Blick bei der ab- 
schließenden Begründung eines neuen Modelltyps grundrechtlich-funktionaler Legitimation (S. 560ff.). Als Begründung bietet der Autor hier den besonderen Bezug der geschützten und zu gewährleistenden Grundrechte zum Prozess der "Willensbildung des Volkes“ und der hierzu erforderlichen Vielfaltsicherung an. In der Konsequenz seines Modells versteht er die besonderen grundrechtlichen Anforderungen an die personelle Besetzung der Privatfunkaufsicht nicht mehr als Kompensation von Legitimationsdefiziten, sondern als unmittelbar aus dem Demokratieprinzip abzuleitende und diesem genügende Bedingungen.

Der Wert des Buches liegt zum einen in der Überwindung einer an Medienverfassungsrecht und Kommunikationswissenschaft orientierten Perspektive, zum anderen in der überbordenden Fülle angesprochener Fragen und einer umfassenden Verarbeitung der einschlägigen Literatur. Das Buch ist ein übersichtlich gegliedertes, akribisch und meinungsfreudig erarbeitetes Kompendium, das viel Aufschluss zu einzelnen Rechtsfragen gibt. Aufs Ganze gesehen hätte allerdings eine stärkere Homogenität und Verzahnung der verwaltungs- und der verfassungsorganisationsrechtlichen Kapitel, an einigen Stellen auch eine Straffung, gut getan. Auch belässt es der Autor bei einer Momentaufnahme, die die Dynamik der Organisation von Medienaufsicht zwar anspricht, aber weder verwaltungs- noch verfassungsrechtlich angemessen verarbeitet. Das Buch liefert damit wichtige Bausteine zum Verständnis der zentralen Medienaufsicht. Doch wird es in Zukunft um sie wohl kaum ruhiger werden.

Margarete Schuler-Harms

\section{Materialien zur interdisziplinären Medienforschung}

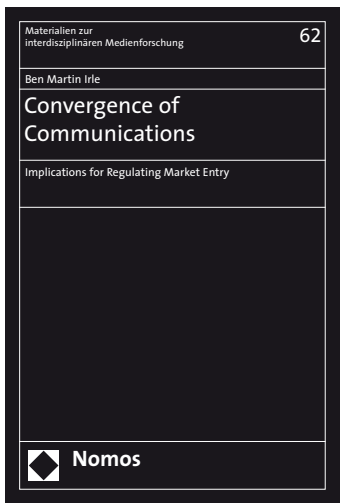

Convergence of Communications Implications for Regulating Market Entry

Von RA Dr. Ben M. Irle, LL.M. 2008, Band 62, ca. 350 S., ca. 69,- $€$, ISBN 978-3-8329-3688-4 Erscheint Dezember 2008

Dem Werk liegt eine Untersuchung zahlreicher Quellen zugrunde und es dient als anschaulicher Überblick über die Entwicklung von Konzentrationsprozessen in der Kommunikation im Allgemeinen und die Folgen für Markterschließungsregelungen im Speziellen.

Bitte bestellen Sie im Buchhandel oder 